\title{
L-arginine supplementation does not improve muscle function during recovery from resistance exercise
}

\begin{tabular}{|c|c|}
\hline Journal: & Applied Physiology, Nutrition, and Metabolism \\
\hline Manuscript ID & apnm-2017-0594.R1 \\
\hline Manuscript Type: & Article \\
\hline Date Submitted by the Author: & 02-Mar-2018 \\
\hline Complete List of Authors: & $\begin{array}{l}\text { Andrade, Walquiria; North University of Paraná (UNOPAR), Center of } \\
\text { Research in Health Sciences } \\
\text { Jacinto, Jeferson; North University of Paraná (UNOPAR), Center of } \\
\text { Research in Health Sciences } \\
\text { da Silva, Douglas; North University of Paraná (UNOPAR), Center of } \\
\text { Research in Health Sciences } \\
\text { Roveratti, Mirela; North University of Paraná (UNOPAR), Center of } \\
\text { Research in Health Sciences } \\
\text { Estoche, José ; North University of Paraná (UNOPAR), Center of Research } \\
\text { in Health Sciences } \\
\text { Oliveira, Douglas ; North University of Paraná (UNOPAR), Center of } \\
\text { Research in Health Sciences } \\
\text { Balvedi, Mario; North University of Paraná (UNOPAR), Center of Research } \\
\text { in Health Sciences } \\
\text { da Silva, Rubens; North University of Paraná (UNOPAR), Center of } \\
\text { Research in Health Sciences } \\
\text { Aguiar, Andreo; North University of Paraná (UNOPAR), Center of Research } \\
\text { in Health Sciences }\end{array}$ \\
\hline Keyword: & $\begin{array}{l}\text { Nutritional ergogenic, skeletal muscle, amino acids, electromyographic, } \\
\text { creatine phosphokinase }\end{array}$ \\
\hline $\begin{array}{r}\text { Is the invited manuscript for } \\
\text { consideration in a Special } \\
\text { Issue? : }\end{array}$ & N/A \\
\hline
\end{tabular}




\section{L-arginine supplementation does not improve muscle function during recovery from resistance exercise}

Andrade, Walquiria Batista ${ }^{1}$; Jacinto, Jeferson Lucas ${ }^{1}$; da Silva, Douglas Kratki ${ }^{1}$; Roveratti, Mirela Casonato ${ }^{1}$; Estoche, José Maria ${ }^{1}$; Oliveira, Douglas Benedito ${ }^{1}$; Balvedi, Mario Carlos Welin ${ }^{1}$; da Silva, Rubens Alexandre ${ }^{1}$; Aguiar, Andreo Fernando ${ }^{1}$

${ }^{1}$ Center of Research in Health Sciences, North University of Paraná (UNOPAR), Londrina, Paraná, Brazil

Corresponding author: Andreo Fernando Aguiar $(\bowtie)$

$\triangle$ Center of Research in Health Sciences, North University of Paraná (UNOPAR), Avenue Paris, 675, Jardim Piza, CEP: 86041-120, Londrina, PR, Brazil. Tel: +55 4399523813, Fax: +55 4333717725, email: afaguiarunesp@gmail.com 


\begin{abstract}
The purpose of this study was to investigate the effects of L-arginine (L-arg) supplementation on muscle recovery process after a single session of high-intensity resistance exercise (RE). 20 healthy young adult $(22.8 \pm 3.4$ y) participants were assigned to two groups $(N=10$ /group $)$ : a placebo-supplement group (PLA) or a L-arginine-supplement group (ARG). The groups completed a session of high-intensity RE (0h), and three subsequent fatigue tests sessions (at 24, 48 and $72 \mathrm{~h}$ post-exercise) to assess the time course of muscle recovery. During the tests sessions, we assessed the following dependent variables: number of maximum repetitions, electromyographic signal (i.e., root mean square [RMS] and median frequency slope [MF]), muscle soreness and perceived exertion, as well as blood levels of creatine kinase (CK) and lactate, and testosterone:cortisol ratio. Number of maximum repetitions increased at 48 and $72 \mathrm{~h}$ post-exercise in both groups (time $P<0.05$ ). CK levels and muscle soreness increased at $24 \mathrm{~h}$ post-exercise, which progressively returned to baseline at $72 \mathrm{~h}$ post-exercise in both gropus (time $P<0.05)$. Lactate levels increased immediately post-exercise, which reduced at $24 \mathrm{~h}$ postexercise in both groups (time $P<0.05$ ). Testosterone:cortisol ratio, RMS and MF slope remained unchanged during recovery period in both groups (time $P>0.05)$. No significant $(P>0.05)$ group x time interaction was found for all dependents variables during the recovery period. In conclusion, our data indicate that L-arg supplementation does not improve muscle recovery process following a high-intensity RE session in young adult individuals.

Keywords: Nutritional ergogenic, skeletal muscle, amino acids, electromyographic, creatine phosphokinase
\end{abstract}




\section{Introduction}

Amino acids are among the most common nutritional supplements used by athletes and recreationally active adults to improve physical performance and muscle recovery (Yavuz et al. 2014). In particular, an over-the-counter supplement called L-arginine (L-arg) has received special attention in last two decades due to its probable ergogenic effects (Àlvares et al. 2011). L-arg is a semi-essential amino acid produced endogenously via intestinal-renal axis [for more details see Wu et al. 2009)]. Briefly, L-arg is synthesized from glutamine, glutamate and proline in the mitochondria of intestinal enterocytes, then released into the bloodstream, and taken up primarily by kidneys for de-novo L-arg production (Crenn and Cynober, 2010). Besides the kidneys, L-arg is also converted into L-arg in others cell types, including adipocytes, endothelial cells, enterocytes, macrophages, neurons, and myocytes (Wu and Morris Jr. 1998), evidencing the biological importance of L-arg in multiple body systems.

The plasma L-arg level is around 40 to $100 \mu \mathrm{mol} \cdot \mathrm{L}^{-1}$ in healthy individuals (Durante W et al. 2007; Àlvares et al. 2012b). Nevertheless, the Exogenous L-arg supplementation (6-10g) is able to increase plasma L-arg levels by around $330-335 \%$ (peak concentration $\left[\mathrm{C}_{\max }\right]$ of $310 \pm$ $152 \mu \mathrm{mol} / \mathrm{L})$ (Bode-Böger et al. 1998; Tangphao et al. 1999), within 1 hour post oral ingestion (fasted state) (Tangphao et al. 1999). This increase L-arg bioavailability has been reported to improve the blood perfusion in the active muscle during exercise (Àlvares et al. 2011; Àlvares et al. 2012b), enhance the clearance of waste-product such as lactate and ammonia (Schaefer et al. 2002), and augment physical performance (Bailey et al. 2010; Pahlavani et al. 2017).

These positive effects are supposedly attributed to an increase in nitric oxide (NO - a potent vasodilator agent) production, since that L-arg is the only substrate for NO synthesis (Àlvares et al. 2012a). The NO-induced vasodilation could increase blood flow and theoretically 
promote higher nutrient and oxygen delivery to the active muscles during exercise, thereby enhancing muscle function and recovery. Besides NO production, L-arg has also been shown to improved muscle adaptations (i.e., enhance myotube density, total nuclei number, and nuclear fusion index) (Long et al. 2006), and increase synthesis of creatine (Durante et al. 2007; Chiarla et al. 2006) - a amino acid involved in multiple biological (i.e., antioxidant and inflammatory) (Fang et al. 2002; Bassit et al. 2008) and metabolic (i.e., glucose metabolism, and ATP resynthesis) (Casey and Greenhaff 2000; Gualano et al. 2008) functions.

Based on the aforementioned mechanisms, it is postulated that L-arg supplementation may improve muscle performance and recovery by increasing nutrients and oxygen delivery to the active muscles, improving clearance of waste-product, and increasing rate of protein synthesis. Despite an abundance of studies on physiological properties and ergogenic effects of L-arg supplementation (Bailey et al. 2010; Àlvares et al. 2012b; Yavuz et al. 2014), no study to date has examined whether L-arg supplementation could improve the muscle function during recovery from a session of high-intensity RE.

The purpose of this study was to investigate the effects of free L-arg supplementation on muscle function during the recovery from a single session of high-intensity RE in untrained young adult individuals. Based on the physiological properties and beneficial effects of L-arg on blood perfusion, protein synthesis, and performance (Bailey et al. 2010), we hypothesized that free L-arg supplementation would enhance muscle function during recovery process from RE by improving muscle functional, metabolic, anabolic, and physiological responses. 


\section{Materials and methods}

\section{Participants}

Twenty-four healthy, recreationally active individuals were recruited from a university population, and 20 completed the study ( 4 withdrew by factors not related to the study). Descriptive characteristics of the participants are presented in Table 1. An a priori power analysis was conducted $\left(\mathrm{G}^{*}\right.$ Power v. 3.0.1) for an $\mathrm{F}$ test (repeated measures, within-between interaction factors for four-time points). On the basis of a statistical power $(1-\beta)$ of 0.80 , a moderate effect size (0.5), and an overall level of significance of 0.05 , least 9 participants were required in each group. Participants were excluded if they: (1) were vegetarian or smoker, (2) had ingested any ergogenic supplement or anabolic steroids for the 6 months prior to the start of study, (3) were taking any medication that could affect muscle recovery or the ability to train intensely, (4) had participated in a exercise training program (more than 2 days per week, for 6 months prior to the beginning of the study), (5) were unable to provide a detailed description of their lifestyle and daily food intake, and (6) did not have medical approval to perform physical exercise. All participants were informed of the procedures, risks, and benefits of the investigation and signed an informed consent document approved by the Institutional Review Board of the University (protocol no: 56725616.3.0000.0108). All procedures were carried out following the principles of the Declaration of Helsinki of 1975, revised in 2008.

\section{Experimental design}

A double-blind, placebo-controlled, parallel-groups design with repeated measures was performed to examine the effects of L-arg supplementation on the time course of muscle recovery after a single session of high-intensity RE in young individuals (Fig. 1). The participants were matched according to sexes and 1RM strength and then randomly assigned to 
two groups ( $N=10$ /group; 7 men and 3 women in each group): L-arginine (ARG) and placebo (PLA). The participants reported to the laboratory four times (e.g., visit 1, visit 2, visit 3, and visit 4) after completed 3 sessions of familiarization and 4 sessions of one-repetition maximum (1RM) tests leg press and hack squat exercises. On visit 1, the participants underwent a single session of high-intensity RE $(0 \mathrm{~h})$ involving leg press and hack squat exercises in order to maximize recruitment of quadriceps muscle. On visit 2, 3 and 4, the participants were submitted to 3 sessions (at 24, 48 and $72 \mathrm{~h}$ after RE session, respectively) of fatigue tests in the leg press to assess the time course of muscle recovery. RE session and fatigues tests were performed 60 minutes after supplementation. In each session of the fatigue test, functional (i.e., number of maximum repetitions, muscle soreness, and perceived exertion), metabolic (i.e., levels of CK and lactate), anabolic (i.e., testosterone:cortisol ratio), and physiological (i.e., RMS and MF slope) indicators of muscle recovery were assessed. Moreover, all participants completed a 3-day dietary intake record during the study period in order to monitor any influence of diet.

We used a two-group design because of its advantages over a crossover design. Parallel groups designs are not influenced by potential carry-over effects (influence from one treatment to another). Moreover, crossover studies require a long wash-out period between treatments, thereby increasing the possibility of participant's dropout. We ensured that the experimental approach used in the present study provides an effective way to investigate the effects of ARG supplementation on muscle recovery in young adult individuals.

\footnotetext{
** Insert Figure 1 near here **
} 


\section{Familiarization protocol}

Before visit 1, all participants completed 3 sessions of familiarization with leg press and hack squat exercises in order to minimize any potential learning effects and establish the reliability of the testing protocols (Fig. 1). The protocol consisted of 3 sets of 8-12 repetitions with 2 min rest between the sets and exercises. Qualified personnel individually supervised each participant during the familiarization period. All sessions of familiarization were performed at the same location, between 8:00 and 10:00.

\section{Determination of one-repetition maximum load}

Before visit 1, all participants completed 4 sessions of 1RM tests $(2$ sessions for each exercise leg press and hack squat), using a standard testing protocol as previously documented elsewhere (Aguiar et al. 2015). The 1RM test was preceded by a set of warm-up exercise ( $\sim 15$ repetitions) at approximately $50 \%$ of the load to be used in the first attempt of the 1 RM test. After 2 min of rest, the $1 \mathrm{RM}$ attempts were performed with a progressively increasing load (5-10\%) for each attempt, and were separated by 4 - to 5-min rest intervals to allow adequate recovery. Only 3 attempts were allowed in each testing session. The exercises were standardized and continuously monitored by the same experienced rater in an attempt to assure the data quality and determine the 10RM within 3 attempts. 1RM was defined as the greatest load lifted through a full range of motion before 2 failed attempts at a given load. Verbal encouragement was provided during all $1 \mathrm{RM}$ attempts. The intraclass correlation coefficients (ICC) test-retest were $\geq 0.94$ for each $1 \mathrm{RM}$ test, indicating the elimination of the learning curve for the participants. All session of 1RM tests were performed at the same location, between 8:00 and 10:00. 


\section{Resistance exercise}

During visit 1, participants were submitted to a session of high-intensity RE (3 sets of 8-12 repetitions at $70 \%$ of $1 \mathrm{RM}$, with 2 min rest between sets and exercises), involving the leg press and hack squat exercises (bilateral) (Nakagym equipment, São Paulo, Brazil). The cadence of muscle action was $1 \mathrm{~s}$ concentric: $2 \mathrm{~s}$ eccentric according metronome. This protocol was designed to maximize the recruitment of quadriceps muscle, and the training stimulus was similar to a session of conventional hypertrophic RE for novice individuals (ACSM, 2009). RE session began with general (moderate walking on treadmill for $10 \mathrm{~min}$ ) and specific (1 set of 12 repetitions with a self-selected load) warm-up exercises for quadriceps muscle. Qualified personnel supervised each participant individually during every workout. The RT sessions were performed at the same location, between 8:00 and 10:00.

\section{Supplementation protocol}

During all visits, the participants ingested an identical looking and equivalent amount $(6 \mathrm{~g})$ of L$\arg (100 \%$ pure $)$ or placebo (cornstarch) dissolved in water $(200 \mathrm{ml})$, in a double-blind and randomized manner. The supplements were analyzed for purity and validated prior to the study. To ensure the double-blind design, an individual who was not involved in the study was responsible for placing the supplements into bags and labeling with the participants' names according to the randomization list. We chose to provide $6 \mathrm{~g}$ of L-arg because such as dose would be safe and well tolerated when orally consumed (Bode-Böger et al. 1998), and has been reported to increase blood flow (Àlvares et al. 2012b) and vasodilatation (Bode-Böger et al. 1998 ) in humans. The supplementation was performed 60 minutes before the RE session and fatigue tests, based on the following pharmacokinetics properties of L-arg: it has been reported 
that the maximal concentration of plasma L-arg is reached within 1 hour post oral ingestion (fasted state) (Tangphao et al. 1999; Liu et al. 2009).

\section{Nutrient intake}

Participants completed a 3-day dietary intake record during the study period. The macronutrient composition of the diets was calculated using software for nutritional assessment (Avanutri, version 3.1.4, Rio de Janeiro-RJ, Brazil). Participants were instructed to duplicate their food intake for the $24 \mathrm{~h}$ proceeding each session, and to refrain from any strenuous activity during experimental period. The participants were also instructed to report any adverse events from the supplements. No discomfort or adverse effects were reported after L-arg ingestion.

\section{Perceived exertion}

Rating of perceived exertion (RPE) was measured immediately after muscular fatigue tests (visits 2, 3 and 4) using the OMNI-RES scale (Robertson et al. 2003). The participants were instructed to report the perceived exertion value indicating a number of the OMNI-RES scale (0 "no effort" and 10 "maximal effort") that best represented their overall muscular effort (Robertson et al. 2003; Marcora, 2009]. The score was the value (0-10) reported in OMNI-RES scale.

\section{Muscle Soreness}

Muscle soreness was measured before muscular endurance tests (visits 2, 3 and 4) using a visual analog scale (VAS). The VAS consists of a 10-cm line whose end points were labeled with "no pain" (left) and "unbearable pain" (right). The participants were instructed to palpate their quadriceps muscle and mark a vertical line at a scale point that best represented their rating of momentary soreness. The score was the distance (in $\mathrm{cm}$ ) from the left side of the scale to the point marked (Mattacola et al. 1997). 


\section{Muscular endurance tests and electromyographic (EMG) signal recordings}

During visits 2, 3 and 4, the participants were submitted to a muscular fatigue test (1 set at $60 \%$ of 1RM until failure) in the leg press exercise (Fig. 1) in order to examine the muscle function (i.e., number of maximum repetitions) during recovery. During the leg press test, surface EMG signals were recorded from the vastus lateralis (VL) muscle using a pre-amplified (gain: 1000) active bipolar surface electrode (Model EMG System Brazil Ltda, São José dos Campos, São Paulo, Brazil) at a sampling rate of $2000 \mathrm{~Hz}$. The participants' skin was prepared by removing the superficial dead skin and was sterilized with an alcohol swab. The electrode was placed on a location near the center of the belly of the muscle according to the SENIAM (Surface EMG for Non-Invasive Assessment of Muscles) recommendations and from our previous work (Aguiar et al. 2015), and the reference electrode was fixed at the right styloid process. The EMG signals were filtered with a band-pass digital filter between 10 and $500 \mathrm{~Hz}$ to remove high frequency noise as well as low-frequency movement.

To determine the muscle activation and fatigue, two EMG parameters were computed: Root mean square (RMS) and Median frequency (MF). RMS corresponds to the muscular activation of the second and before last contractions of the endurance test (e.g., to avoid the acceleration and the deceleration portions of the concentric leg contractions during the extension phase of movement). This parameter was computed by a moving RMS method executed on successive $250 \mathrm{~ms}$ (512 points) time-series windows (50\% overlap) to obtain the RMS average values during the entire leg press exercise. MF corresponds to muscle fatigue from the magnitude of the electromyographic spectral content evaluated by the MF value of the power spectra (Short-fast Fourier transform, Hanning window processing) during successive time windows (50\% overlap) of $250 \mathrm{~ms}$ for the total time of the fatigue test. Least squares linear 
regression analysis was applied to the MF time series to calculate the rate of decline in MF over time (MF/time slope as a muscle fatigue index), as supported by previous studies (Larivière et al. 2002; Kienbacher et al. 2014; da Silva et al. 2015). All EMG signals and estimates (RMS and $M F$ ) were processed using MATLAB sub-routines for data computation from subsequent analysis (Version 8.0, Mathworks ${ }^{\circledR}$, South Natick, MA, USA).

\section{Blood collection and analysis}

Blood samples were collected at pre- and post-training, and immediately after muscular fatigue tests (visits 2, 3 and 4) for analyses of CK, lactate, glucose, cholesterol, testosterone, and cortisol concentrations (Fig. 1). The blood samples were allowed to coagulate at room temperature for 60 min and then centrifuged at 2,000 $\mathrm{g}$ for $15 \mathrm{~min}$, and the serum was frozen at $-80^{\circ} \mathrm{C}$ until analysis. All analyses were performed in a laboratory equipped with automated systems using commercial kits for chemiluminescence (testosterone and cortisol), kinetic (CK and lactate), and enzymatic (cholesterol, glucose) techniques.

\section{Statistical analyses}

Data are expressed as means $\pm \mathrm{SD}$. The normality and homogeneity for outcome measures were tested using the Shapiro-Wilk's and Levene's tests, respectively. Independent variables included the supplementation protocol (i.e., PLA vs. ARG) and time (i.e., 24, 48, and 72h). Dependent variables included number of maximum repetitions, perceived exertion, muscle soreness, EMG signals (RMS and MF), and blood analyses (i.e., glucose, cholesterol, CPK, lactate, insulin, testosterone and lactate). Baseline characteristics and food intake between groups were compared using an unpaired $t$-test. Two-way (group $\mathrm{x}$ time) ANOVA with repeated measures was used to evaluate the data across time and between groups for all independent variables. Violation of sphericity was adjusted by Greenhouse-Geisser. When significant differences were confirmed 
with ANOVA, multiple comparisons testing were performed using Tukey post hoc analysis to identify these differences. The significance level was set at $P \leq 0.05$. Statistical analyses were performed using SPSS statistical analysis software (SPSS version 20.0; Chicago, IL, USA).

Conventional statistics ( $P$-value) are commonly used to test (and dismiss) a 'null hypothesis', which generally states that there is no difference between two groups. A $P$-value of 0.05 or less is generally taken to mean that a finding is statistically significant (rejecting a null hypothesis) and warrants publication. But that is not necessarily true. We can disprove results only in pure mathematics, not in real life, in which some useful effects may not be statistically significant and some statistically significant effects may not be useful. Therefore, the effect size (ES) and confidential interval (CI) have been recommended as a more appropriate analysis for evidence the magnitude of an intervention (treatment effect) (Nakagawa and Cuthill, 2007; Page, 2014), due their biological importance to make clinical decisions (Page, 2014), and elimination of confounding factors such as sample size and measure variability (Battehham and Hopkings, 2006; Wilkinson, 2014; Hopkings et al. 2009). Thus, we analyzed ES and 95\% CI (Cohen et al. 1977) to ensure a more realistic biological interpretation of data, and to provide practical information on the magnitude or direction of the difference (treatment effect). 


\section{Results}

Participants characteristics and dietary intake

The ARG and PLA groups had similar $(P>0.05)$ basal characteristics (Table 1$)$. There were no significant $(P>0.05)$ differences between ARG and PLA groups in the carbohydrate (ARG: 3.2 \pm 1.3 vs. PLA: $3.6 \pm 1.3, P>0.05$ ), protein (ARG: $1.4 \pm 0.7$ vs. PLA: $1.5 \pm 0.6, P>0.05$ ), lipids (ARG: $1.1 \pm 0.5$ vs. $1.0 \pm 0.5, P>0.05$ ) daily intake, and total kilocalories (ARG: $2076.5 \pm$ 879.9 vs. PLA: $2115.4 \pm 525.2, P>0.05)$ during the study period, and both groups had an adequate macronutrients intake according to the recommendations proposed by the American College of Sports Medicine (Thomas et al. 2006).

\section{** Insert Table 1 near here **}

Perceived exertion and number of maximum repetitions in the fatigue tests during recovery. Both ARG and PLA groups achieved maximum effort in the fatigue tests (mean OMNI scale; ARG: $9.4 \pm 0.8 v s$. PLA: $9.3 \pm 0.9 ; P>0.05)$, indicating that protocol was reliable for maximize the muscle recruitment. There was no significant $(P>0.05)$ group $\mathrm{x}$ time interaction, but a significant main effect for time $(P<0.05)$ indicated an increase in number of maximum repetitions at 48 and $72 \mathrm{~h}$ post-exercise in both groups (Fig. 2). AUC over 3 days of recovery was similar $(P>0.05)$ between groups (Fig. 2; Left upper figure). The ES (95\% CI) for total repetitions over the 3 days of recovery is shown in Fig. 2 (Right upper figure). The data show no positive effect in favor of ARG group.

\section{** Insert Figure 2 near here **}

CK and lactate blood levels after fatigue test during recovery. There was no significant group $\mathrm{x}$ time interaction $(P>0.05)$, but a significant main effect for time $(P<0.05)$ indicated an increase in serum CK levels (Fig. 3A) at 24 h post-exercise (ARG: +42 vs. PLA: $+45 \%, P>0.05$ ) which 
remained elevate at $48 \mathrm{~h}(\mathrm{ARG}:+41$ vs. $+31 \%, P>0.05)$ in both ARG and PLA groups (Fig. 4A), and gradually returned to baseline at $72 \mathrm{~h}$ post-exercise. Plasma lactate levels increased (time $P<$ 0.05) immediately post-exercise (Fig. 3B), which reduced at $24 \mathrm{~h}$ post-exercise (time $P<0.05$ ), with no differences between CM and PL conditions (group x time $P>0.05$ ). AUC for CK (Fig. 3A; Left upper figure) and lactate (Fig. 3B; Left upper figure) over 3 days of recovery were similar $(P>0.05)$ between groups. The ES $(95 \% \mathrm{CI})$ for CK (Fig. 3A; Right upper figure) and lactate (Fig. 3B; Right upper figure) over the 3 days of recovery show no positive effect in favor of ARG group compared to PLA group.

\section{** Insert Figure 3 near here **}

Muscle Soreness during recovery. There was no significant group $\mathrm{x}$ time interaction $(P>0.05)$, but a significant main effect for time $(P<0.05)$ indicated a decrease in perceived intensity of muscle soreness from $24 \mathrm{~h}$ (ARG: $4.5 \pm 2.5$ vs. PLA: $4.3 \pm 2.8, P>0.05)$ and $48 \mathrm{~h}(\mathrm{ARG}: 5.3 \pm$ $3.3 v s$. PLA: $5.8 \pm 2.1, P>0.05)$ to 72 h post-exercise (ARG: $3.9 \pm 2.5$ vs. PLA: $3.2 \pm 1.7, P>$ 0.05) in both ARG and PLA groups.

Testosterone:cortisol ratio after fatigue test during recovery. Testosterone:cortisol ratio (Figure 4) did not differ between ARG and PLA conditions during recovery period at 24, 48, and $72 \mathrm{~h}$ post-exercise (treatment $\mathrm{x}$ time $P>0.05$; time $P>0.05$ ). AUC over 3 days of recovery was similar $(P>0.05)$ between groups (Fig. 4; Left upper panel). The ES (95\% CI) for testosterone:cortisol ratio over the 3 days of recovery is shown in Fig. 4 (Right upper panel). The data show no positive effect in favor of ARG group compared to PLA group.

\section{** Insert Figure 4 near here **}

EMG signal in the fatigue tests during recovery. There was no significant main effect for time $(P$ $>0.05)$ or group $x$ time interaction $(P>0.05)$ in RMS (Fig. 5A) and MF slope (Fig. 5A and B, 
respectively) in the fatigue tests during the recovery period $(24,48$, and $72 \mathrm{~h}$ post-training). The mean RMS (ARG $209.8 \pm 167.8 v s$. PLA: $212.2 \pm 100.0 ; P>0.05$ ) and MF slope (ARG: -0.044 $\pm 0,029$ vs. PLA: $-0.050 \pm 0.024 ; P>0.05$ ) over the 3 days of recovery were similar between ARG and PLA groups. AUC for RMS and MF slope (Fig. 5A and B, respectively; Left upper panel) over 3 days of recovery were similar $(P>0.05)$ between ARG and PLA groups. The ES (95\% CI) for RMS and MF slope over the 3 days of recovery are shown in Fig. 5A and B, respectively (Right upper panel). The data show a positive effect in the MF slope in favor of ARG group.

** Insert Figure 5 near here ** 


\section{Discussion}

To our knowledge, this is the first study to examine the effects of free L-arg supplementation on the time course of muscle recovery after a single session of conventional high-intensity RE in untrained young adult individuals. Based on the physiological properties and beneficial L-arg effects on blood perfusion, protein synthesis, and performance (Bailey et al. 2010; Àlvares et al. 2011; Àlvares et al. 2012b), we hypothesized that free L-arg supplementation would enhance muscle recovery process post-exercise by improving mechanical, physiological, metabolic, anabolic responses. In contrast to the hypothesis, we observed that free L-arg supplementation (6 $\mathrm{g}$ at 60 min pre-workout) does not improve the major functional (i.e., number of maximum repetitions, muscle soreness, and perceived exertion), metabolic (i.e., CK, and lactate), anabolic (i.e., testosterone:cortisol ratio), and physiological (i.e., RMS and MF signal) indicators of muscle recovery in untrained young adult men.

Given that L-arg is the main substrate for the NO synthesis, it has been postulated that Larg supplementation may increase NO production and, consequently, improve the vasodilation and blood perfusion in the active muscle during exercise (Àlvares et al. 2011; Àlvares et al. 2012b). As a result, L-arg supplementation could enhance the clearance of waste-product such as lactate and ammonia (Schaefer et al. 2002), and promote higher nutrient and oxygen delivery to the muscles during exercise, thereby enhancing muscle performance and recovery. Nevertheless, we have shown that L-arg supplementation does not promote any improvements in markers of muscle function (i.e., number of maximum repetitions, muscle soreness, and perceived exertion) during recovery from high-intensity $\mathrm{RE}(24,48$, ad $72 \mathrm{~h}$ post-exercise). Consistent with our results, previous performance studies have shown that free L-arg supplementation or combined with alpha ketoglutarate (AAKG) had no beneficial effect on maximal dynamic strength (1RM) 
(Liu et al. 2009), isokinetic strength (Àlvares et al. 2012a), muscle endurance (i.e., repetition performed) (Greer et al. 2011; Wax et al. 2013) and performance in intermittent anaerobic exercise in trained individuals (Wax et al. 2012). There are two possibilities that might explain this lack of a positive effect of L-arg supplementation on muscle recovery and performance. First, L-arg supplementation may be ineffective to increase NO production and, consequently, muscle blood flow at rest and exercise conditions in healthy individuals. This hypothesis is supported by several studies involving healthy individuals that showed no additional effect of Larg supplementation (range: $6-10 \mathrm{~g}$ ) on $\mathrm{NO}$ production (measured by $\mathrm{NO}_{2}{ }^{-}$and $\mathrm{NO}_{3}{ }^{-}$) (Blum et al. 2000; Evans et al. 2004; Àlvares et al; 2012a; Liu et al. 2009; Koppo et al. 2009; Tang et al. 2011) and blood flow (Blum et al. 2000; Tang et al. 2011; Aguiar et al. 2016), despite increases in plasma L-arg concentration (Blum et al. 2000; Evans et al. 2004; Liu et al. 2009; Tang et al. 2011; Àlvares et al; 2012a). This is consistent with fact that physiological concentrations of Larg (range: 40 to $100 \mu \mathrm{mol} \cdot \mathrm{L}^{-1}$ ) in healthy individuals are sufficient to saturate endothelial nitric oxide synthase, which is $\sim 2-20 \mu \mathrm{mol} \cdot \mathrm{L}^{-1}$ (Durante et al. 2007), resulting in no additional effect on NO production. Therefore, in light of these evidences, it seems unlikely that L-arg supplementation may improve muscle recovery and function by mechanisms related to NOinduced-increase in vasodilatation and muscle blood flow (e.g., increasing nutrients and oxygen delivery to the active muscles, and improving clearance of waste-product, such as ammonia and lactate). Our results confirm this hypothesis by showing for the first time that L-arg supplementation does not improve muscle function and markers of muscle fatigue (i.e., lactate and perceived exertion) and damage (i.e., CK and muscle soreness) during recovery from highintensity RE in healthy young adult individuals. It is important to note that a dose of $6 \mathrm{~g}$ is sufficient to significantly increase plasma L-arg levels, without any additional effect on NO 
production (Àlvares et al; 2012a; Liu et al. 2009). Thus, the lack of a positive effect of L-arg supplementation on muscle recovery from RE does not necessarily indicate that our participants did not experience an increase in the plasma L-arg levels.

Second, the lack of a positive effect of L-arg supplementation on muscle recovery from RE may be due to its inability to improve the muscle anabolic and regenerative profile of skeletal muscle (e.g., increase anabolic hormones and/or protein synthesis rate). This hypothesis is consistent with previous studies that reported an increased physical performance (e.g., time to exhaustion) (Bailey et al. 2010; Yavuz et al. 2014), but no improvement on muscle protein synthesis rate (Tang et al. 2011) after L-arg supplementation in humans. Additionally, L-argaspartate supplementation has failed to affect anabolic parameters (e.g., concentration of insulin, growth hormone [GH], and testosterone) after exercise (Colombani et al. 1999; Abel et al. 2005). This lack of L-arg effects on muscle anabolism and regeneration markers (Colombani et al. 1999; Abel et al. 2005; Tang et al. 2011) was supported by the absence of differences in anabolic parameters (i.e., testosterone:cortisol ratio) between ARG and PLA groups in our study. Therefore, it is likely that the inability of L-arg supplementation to improve anabolic factors resulted in no beneficial effect on muscle function (i.e., number of maximum repetition), fatigue (i.e., lactate and perceived exertion), and damage (i.e., $\mathrm{CK}$ and muscle soreness) markers during recovery process from high-intensity RE. It is important to note that some studies (Bailey et al. 2010; Yavuz et al. 2014) have shown a beneficial effect of L-arg supplementation on exercise performance when muscle was tested in normal conditions (without any exercise-induced damage); nevertheless, our results show that L-arg supplementation does not improve muscle function during recovery from high-intensity RE (when muscle is exposed to a high degree of fatigue and damage). One possible explanation for these results is that the beneficial effects of L- 
arg supplementation on exercise performance are less than the impairment in the muscle function after a high-intensity RE session, resulting in no significant improvement in the recovery process.

Additionally, we showed no differences in electromyographic (EMG) indicators of muscle activation (RMS) and fatigue (MF) between ARG and PLA groups during recovery period. To our knowledge, this the first study to examine the effects of L-arg supplementation on EMG indicators of muscle activation (RMS) and fatigue (MF slope) during recovery from highintensity RE. Considering that a decline in MF and increase in RMS are typically associated with muscle fatigue during isometric and dynamic contractions (Adam and De Luca 2003; Arab et al. 2007; da Silva et al. 2008), we expected an inverse effect of L-arg supplementation on these factors in the fatigue test during recovery period. However, no differences were observed in RMS and MF slope between the ARG and PLA groups (despite a beneficial effect in the MF slope in favor of the ARG group), which is in line with the lack of a beneficial effect of L-arg supplementation on muscle function (i.e., number of maximum repetition), fatigue (i.e., lactate and perceived exertion), and damage (i.e., CK) markers. This indicates that L-arg supplementation is not effective in improving the neuromuscular responses during recovery from RE. This result is consistent with no difference in the muscle soreness between ARG and PLA groups during the recovery period. Although muscle soreness may be a poor indicator of exercise-induced muscle damage (Nosaka et al. 2002), it usually reflects muscle fatigue. This supports the findings of the present study that L-arg supplementation does not attenuate muscle fatigue during the course of recovery. Therefore, any possible physiological effects of L-arg supplementation on blood perfusion (Àlvares et al. 2012b), and performance (Bailey et al. 2010; 
Yavuz et al. 2014) may not be sufficient to improve muscle recovery in untrained young adult individuals.

A few limitations of this study must be mentioned. First, we did not analyze plasma NO and arginine concentrations. However, previous studies that used the same L-arg dose (i.e., $6 \mathrm{~g}$ ) showed an increase in plasma arginine concentration (Bode-Böger et al. 1998; Liu et al. 2009; Àlvares et al. 2012a). Second, we did not collect muscle biopsies for analysis of muscle tissue markers of muscle regeneration (e.g. IGF-I, HGF, mTOR, and p70S6k); however, we analyzed the major markers of muscle regeneration (i.e., CPK, insulin, testosterone, and cortisol) as well as functional outcomes measures (i.e., number of maximum repetitions, perceived exertion, muscle soreness, and EMG signs) function. Finally, we did not standardize the participants' diet; however, we did instruct participants to duplicate their food intake for the $24 \mathrm{~h}$ proceeding each session.

In conclusion, our data indicate that L-arg supplementation (single $6 \mathrm{~g}$ dose at $60 \mathrm{~min}$ pre-workout) does not improve muscle recovery from a single session of high-intensity RE in untrained young adult individuals. Therefore, it is still premature to recommend free L-arg supplementation as an ergogenic aid to improve muscle recovery after RE. Future studies are required to assess the effects of L-arg supplementation in others populations (e.g., elderly and/or women) with different training status (e.g., recreational practitioners and/or athletes).

\section{Acknowledgement}

We address special thanks to all the participants for their engagement in this study. Funding for the study was provided by North University of Paraná (UNOPAR).

\section{Conflict of interest}

No conflicts of interest, financial, or otherwise, are declared by the author(s). 


\section{References}

Abel, T., Knechtle, B., Perret, C., Eser, P., von Arx, P., and Knecht, H. 2005. Influence of chronic supplementation of arginine aspartate in endurance athletes on performance and substrate metabolism - a randomized, double-blind, placebo-controlled study. Int. J. Sports. Med. 26(5): 344-349. doi: 10.1055/s-2004-821111.

Adam, A., and De Luca, C.J. 2003. Recruitment order of motor units in human vastus lateralis muscle is maintained during fatiguing contractions. J. Neurophysiol. 90(5): 2919-2927. doi: 10.1152/jn.00179.2003.

Aguiar, A.F., Buzzachera, C.F., Pereira, R.M,, Sanches, V.C., Januário, R.B., da Silva, R.A., et al. 2015. A single set of exhaustive exercise before resistance training improves muscular performance in young men. Eur. J. Appl. Physiol. 115(7): 1589-1599. doi: 10.1007/s00421-015$3150-8$

Aguiar, A.F., Balvedi, M.C., Buzzachera, C.F., Altimari, L.R., Lozovoy, M.A., Bigliassi, M., et al. 2016. L-Arginine supplementation does not enhance blood flow and muscle performance in healthy and physically active older women. Eur. J. Nutr. 55(6): 2053-62. doi: 10.1007/s00394015-1019-6.

Àlvares, T.S., Meirelles, C.M., Bhambhani, Y.N., Paschoalin, V.M., and Gomes, P.S. 2011. Larginine as a potential ergogenic aid in healthy subjects. Sport. Med. 41(3): 233-248. doi: 10.2165/11538590-000000000-00000.

Àlvares, T.S., Conte-Junior, C.A., Silva, J.T., and Paschoalin, V.M. 2012a. Acute L-Arginine supplementation does not increase nitric oxide production in healthy subjects. Nutr. Metab. (Lond). 9(1): 54. doi: 10.1186/1743-7075-9-54. 
Àlvares, T.S., Conte, C.A., Paschoalin, V.M., Silva, J.T., Meirelles, Cde M., Bhambhani, Y.N., et al. 2012b. Acute L-arginine supplementation increases muscle blood volume but not strength performance. Appl. Physiol. Nutr. Metab. 37(1): 115-126. doi: 10.1139/h11-144.

American Collge of Sports Medicine position stand (ACSM). 2009. Progression models in resistance training for healthy adults. Med. Sci. Sports. Exerc. 41(3): 687-708. doi: 10.1249/MSS.0b013e3181915670.

Arab, A.M., Salavati, M., Ebrahimi, I., and Ebrahim Mousavi, M. 2007. Sensitivity, specificity and predictive value of the clinical trunk muscle endurance tests in low back pain. Clin. Rehabil. 21(7): 640-647. doi:10.1177/0269215507076353.

Bailey, S.J., Winyard, P.G., Vanhatalo, A., Blackwell, J.R., DiMenna, F.J., Wilkerson, D.P., et al. 2010. Acute L-arginine supplementation reduces the $\mathrm{O} 2$ cost of moderate-intensity exercise and enhances high-intensity exercise tolerance. J. Appl. Physiol. 109(5): 1394-1403. doi: 10.1152/japplphysiol.00503.2010.

Bassit, R.A., Curi, R., and Costa Rosa, L.F. 2008. Creatine supplementation reduces plasma levels of pro-inflammatory cytokines and PGE2 after a half-ironman competition. Amino Acids. 35(2): 425-431. doi: 10.1007/s00726-007-0582-4.

Batterham, A.M., and Hopkins, W.G. 2006. Making meaningful inferences about magnitudes. Int. J. Sports. Physiol. Perform. 1(1): 50-57. PMID: 19114737.

Blum, A., Hathaway, L., Mincemoyer, R., Schenke, W.H., Kirby, M., Csako, G., et al. 2000. Effects of oral L-arginine on endothelium-dependent vasodilation and markers of inflammation in healthy postmenopausal women. J. Am. Coll. Cardiol. 35(2): 271-276. PMID: 10676669.

Bode-Böger, S.M., Böger, R.H., Galland, A., Tsikas, D., Frölich, J.C. 1998. L-arginine-induced 
vasodilation in healthy humans: pharmacokinetic-pharmacodynamic relationship. Br. J. Clin. Pharmacol. 46(5): 489-97. PMID: 9833603.

Casey, A., and Greenhaff, P.L. 2000. Does dietary creatine supplementation play a role in skeletal muscle metabolism and performance? Am. J. Clin. Nutr. 72(2): 607-617. PMID: 10919967.

Chiarla, C., Giovannini, I., and Siegel, J.H. 2006. Plasma arginine correlations in trauma and sepsis. Amino Acids. 30(1): 81-86. doi: 10.1007/s00726-005-0211-z.

Cohen, J. 1977. Statistical power analysis for behavioral sciences, 1st ed.; New York: Academic Press, USA, pp. 490; 9781483276489.

Colombani, P.C., Bitzi, R., Frey-Rindova, P., Frey, W., Arnold, M., Langhans, W., et al. 1999. Chronic arginine aspartate supplementation in runners reduces total plasma amino acid level at rest and during a marathon run. Eur. J. Nutr. 38(6): 263-270. PDIM: 10784382.

Crenn, P., and Cynober, L. 2010. Effect of intestinal resections on arginine metabolism: practical implications for nutrition support. Curr. Opin. Clin. Nutr. Metab. Care. 13(1): 65-69. doi: 10.1097/MCO.0b013e328333c1a8.

da Silva, R.A., Larivière, C., Arsenault, A.B., Nadeau, S., and Plamondon, A. 2008. The comparison of wavelet- and Fourier-based electromyographic indices of back muscle fatigue during dynamic contractions: validity and reliability results. Electroencephalogr. Clin. Neurophysiol. 48(3-4): 147-162. PMID: 18551835.

da Silva, R.A., Vieira, E.R., Cabrera, M., Altimari, L.R., Aguiar, A.F., Nowotny, A.H., et al. 2015. Back muscle fatigue of younger and older adults with and without chronic low back pain using two protocols: A case-control study. J. Electromyogr. Kinesiol. 25(6): 928-936. doi: 
10.1016/j.jelekin.2015.10.003.

Durante, W., Johnson, F.K., and Johnson, R.A. 2007. Arginase: a critical regulator of nitric oxide synthesis and vascular function. Clin. Exp. Pharmacol. Physiol. 34(9): 906-911. doi: 10.1111/j.1440-1681.2007.04638.x

Fang, Y.Z., Yang, S., and Wu, G. 2002. Free radicals, antioxidants, and nutrition. Nutrition. 18(10): 872-879. PMID: 12361782.

Greer, B.K., and Jones, B.T. 2011. Acute arginine supplementation fails to improve muscle endurance or affect blood pressure responses to resistance training. J. Strength. Cond. Res. 25(7): 1789-1794. doi: 10.1519/JSC.0b013e3181e07569.

Gualano, B., Novaes, R.B., Artioli, G.G., Freire, T.O., Coelho, D.F., Scagliusi, F.B., et al. 2008. Effects of creatine supplementation on glucose tolerance and insulin sensitivity in sedentary healthy males undergoing aerobic training. Amino Acids. 34(2): 245-250. doi: 10.1007/s00726007-0508-1.

Hopkins, W.G., Marshall, S.W., Batterham, A.M. and Hanin, J. 2009. Progressive statistics for studies in sports medicine and exercise science. Med. Sci. Sports. Exerc. 41(1): 3-13. doi: 10.1249/MSS.0b013e31818cb278.

Kienbacher, T., Habenicht, R., Starek, C., Mair, P., Wolf, M., Paul, B., et al. 2014. The potential use of spectral electromyographic fatigue as a screening and outcome monitoring tool of sarcopenic back muscle alterations. J. Neuro. Eng. Rehabil. 11: 106. doi: 10.1186/1743-0003-11106.

Koppo, K., Taes, Y.E., Pottier, A., Boone, J., Bouckaert, J., and Derave, W. 2009. Dietary arginine supplementation speeds pulmonary VO2 kinetics during cycle exercise. Med. Sci. 
Sports. Exerc. 41(8): 1626-1632. doi: 10.1249/MSS.0b013e31819d81b6.

Larivière, C., Arsenault, A.B., Gravel, D., Gagnon, D., and Loisel, P. 2002. Evaluation of measurement strategies to increase the reliability of EMG spectral indices to assess back muscle fatigue and recovery. J. Electromyogr. Kinesiol. 12(2): 91-102. PMID: 11955981.

Liu, T.H., Wu, C.L., Chiang, C.W., Lo, Y.W., Tseng, H.F., and Chang, CK. 2009. No effect of short-term arginine supplementation on nitric oxide production, metabolism and performance in intermittent exercise in athletes. J. Nutr. Biochem. 20(6): 462-468. doi: 10.1016/j.jnutbio.2008.05.005.

Long, J.H., Lira, V.A., Soltow, Q.A., Betters, J.L., Sellman, J.E., and Criswell, D.S. 2006. Arginine supplementation induces myoblast fusion via augmentation of nitric oxide production. J. Muscle. Res. Cell. Motil. 27(8): 577-584. doi: 10.1007/s10974-006-9078-1.

Marcora S. 2009. Perception of effort during exercise is independent of afferent feedback from skeletal muscles, heart, and lungs. J. Appl. Phyiology. 106(6): 2060-2062. doi: 10.1152/japplphysiol.90378.2008.

Mattacola, C.G., Perrin, D.H., Gansneder, B.M., Allen, J.D., and Micckey, C.A. 1997. A comparison of visual analog and graphic rating scales for assessing pain following delayed onset muscle soreness. J. Sport. Rehabil. 6(1): 38-46. doi: https://doi.org/10.1123/jsr.6.1.38.

Nakagawa, S., and Cuthill, I.C. 2007. Effect size, confidence interval and statistical significance: a practical guide for biologists. Bio. Rev. Camb. Philos. Soc. 82(4): 591-605. doi: 10.1111/j.1469-185X.2007.00027.x.

Nosaka, K., Newton, M., and Sacco, P. 2002. Delayed-onset muscle soreness does not reflect the magnitude of eccentric exercise-induced muscle damage. Scand. J. Med. Sci. Sports. 12(6): 337- 
346. PMID: 12453160.

Page, P. 2014. Beyond statistical significance: clinical interpretation of rehabilitation research literature. Int. J. Sports. Phys. Ther. 9(5): 726-736. PMID: 25328834.

Pahlavani, N., Entezari, M.H., Nasiri, M., Miri, A., Rezaie, M., Bagheri-Bidakhavidi, M., et al. 2017. The effect of l-arginine supplementation on body composition and performance in male athletes: a double-blinded randomized clinical trial. Eur. J. Clin. Nutr. 71(4): 544-548. PMDI: 28120856.

Robertson, R.J., Goss, F.L., Rutkowski, J., Lenz, B., Dixon, C., Timmer, J., et al. 2003. Concurrent validation of the OMNI perceived exertion scale for resistance exercise. Med. Sci. Sports. Exerc. 35(2): 333-341. doi: 10.1249/01.MSS.0000048831.15016.2A.

Schaefer, A., Piquard, F., Geny, B., Doutreleau, S., Lampert, E., Mettauer, B., et al. 2002. Larginine reduces exercise-induced increase in plasma lactate and ammonia. Int. J. Sports. Med. 23(6): 403-407. doi: 10.1055/s-2002-33743.

Tang, J.E., Lysecki, P.J., Manolakos, J.J., MacDonald, M.J., Tarnopolsky, M.A., and Phillips, S.M. 2011. Bolus arginine supplementation affects neither muscle blood flow nor muscle protein synthesis in young men at rest or after resistance exercise. J. Nutr. 141(2): 195-200. doi: 10.3945/jn.110.130138.

Tangphao, O., Grossmann, M., Chalon, S., Hoffman, B.B., and Blaschke, T.F. 1999. Pharmacokinetics of intravenous and oral L-arginine in normal volunteers. Br. J. Clin. Pharmacol. 47(3): 261-266. PMID: 10215749.

Thomas, D.T., Erdman, K.A., and Burke, L.M. 2016. American College of Sports Medicine Joint Position Statement. Nutrition and Athletic Performance. Med. Sci. Sports. Exerc. 48(3): 543-68. 
doi: 10.1249/MSS.0000000000000852.

Wax, B., Kavazism A,N., Webb, H.E., and Brown, S.P. 2012. Acute L-arginine alpha ketoglutarate supplementation fails to improve muscular performance in resistance trained and untrained men. J. Int. Soc. Sports. Nutr. 9(1): 17. doi: 10.1186/1550-2783-9-17.

Wax, B., Mayo, J.J., Hilton, L.A., Mareio, H.C., Miller, J.D., Webb, H.E., et al. 2013. Acute Ingestion of L-Arginine Alpha-Ketoglutarate Fails To Improve Muscular Strength and Endurance in ROTC Cadets. Int. J. Exerc. Sci. 6(2): 91-97.

Wilkinson, M. 2014. Distinguishing between statistical significance and practical/clinical meaningfulness using statistical inference. Sports. Med. 44(3): 295-301. doi: 10.1007/s40279013-0125-y.

Wu, G., Bazer, F.W, Davis, T.A., Kim, S.W., Li, P., Marc Rhoads, J., et al. 2009. Arginine metabolism and nutrition in growth, health and disease. Amino Acids. 37(1): 153-168. doi: 10.1007/s00726-008-0210-y.

Wu, G., and Morris, S.M. Jr. 1998. Arginine metabolism: nitric oxide and beyond. Biochem. J. 336(1): 1-17. PMID: 9806879.

Yavuz, H.U., Turnagol, H., and Demirel, A.H. 2014. Pre-exercise arginine supplementation increases time to exhaustion in elite male wrestlers. Biol. Sport. 31(3): 187-191. doi: $10.5604 / 20831862.1111436$. 


\section{FIGURE LEGENDS}

Figure 1. Experimental design.

Figure 2. Number of maximum repetitions in the leg press fatigue tests between ARG and PLA groups during $3-\mathrm{d}$ recovery period $(24,48$, and $72 \mathrm{~h}$ post-exercise). Data are means $\pm \mathrm{SD}$. Left upper figure indicates the area-under-curve (AUC) during 3-d recovery period. (Repeatedmeasures ANOVA: ${ }^{\mathrm{a}} P<0.05$ compared to $24 \mathrm{~h} ;{ }^{\mathrm{b}} P<0.05$ compared to $48 \mathrm{~h}$; Group $\mathrm{x}$ time $P>$ 0.05). Right upper figure indicates the effect size (95\% CI) between ARG and PLA groups during 3-d recovery period. Note: The point "0" indicates the PLA group. When CI is further from " 0 " represent more difference between groups (positive or negative), and the CI closer to “ 0 " represent less group difference or effect. When CI contains " 0 " means that there is no effect of treatment or difference between groups. The data show no positive effect in favor of ARG.

Figure 3. Blood CK (A) and lactate (B) levels between ARG and PLA groups at pre- and postexercise, and immediately after fatigue tests during $3-\mathrm{d}$ recovery period $(24,48$, and $72 \mathrm{~h}$ postexercise). Data are means \pm SD. Left upper figure indicates the area-under-curve (AUC) during 3-d recovery period. (Repeated-measures ANOVA: ${ }^{\mathrm{a}} P<0.05$ compared to Pre, Post, and $72 \mathrm{~h} ;{ }^{\mathrm{b}} P$ $<0.05$ compared to Pre; ${ }^{\mathrm{c}} P<0.05$ compared to Pre and Post; Group x time $\left.P>0.05\right)$. Right upper figure indicates the effect size $(95 \% \mathrm{CI})$ between ARG and PLA groups during 3-d recovery period. Note: The point " 0 " indicates the PLA group. When CI is further from " 0 " represent more difference between groups (positive or negative), and the CI closer to " 0 " represent less group difference or effect. When CI contains " 0 " means that there is no effect of treatment or difference between groups. The data show no positive effect in favor of ARG. 
Figure 4. Testosterone:cortisol ratio between ARG and PLA groups at pre- and post-exercise, and immediately after fatigue tests during 3-d recovery period (24, 48, and $72 \mathrm{~h}$ post-exercise). Data are means \pm SD. Left upper figure indicates the area-under-curve (AUC) during 3-d recovery period. (Repeated-measures ANOVA: time $P>0.05$; Group x time $P>0.05$ ). Right upper figure indicates the effect size $(95 \% \mathrm{CI})$ between ARG and PLA groups during 3-d recovery period. Note: The point " 0 " indicates the PLA group. When CI is further from " 0 " represent more difference between groups (positive or negative), and the CI closer to "0" represent less group difference or effect. When CI contains " 0 " means that there is no effect of treatment or difference between groups. The data show no positive effect in favor of ARG.

Figure 5. RMS (A) and MF slope (B) values between ARG and PLA groups in the leg press fatigue test during 3 -d recovery period $(24,48$, and $72 \mathrm{~h}$ post-exercise). Data are means $\pm \mathrm{SD}$. Left upper figure indicates the area-under-curve (AUC) during 3-d recovery period. (Repeatedmeasures ANOVA: time $P>0.05$; Group $\mathrm{x}$ time $P>0.05$ ). Right upper figure indicates the effect size $(95 \% \mathrm{CI})$ between ARG and PLA groups during 3-d recovery period. Note: The point " 0 " indicates the PLA group. When CI is further from " 0 " represent more difference between groups (positive or negative), and the CI closer to "0" represent less group difference or effect. When CI contains " 0 " means that there is no effect of treatment or difference between groups. The data show a positive effect in the MF slope in favor of ARG group. 


\section{TABLES}

Table 1. Participants Characteristics.

\begin{tabular}{lcc}
\hline & ARG $(N=10)$ & PLA $(N=10)$ \\
\hline Age $(\mathrm{y})$ & $23.0 \pm 4.0$ & $22.5 \pm 3.3$ \\
Height $(\mathrm{cm})$ & $171.9 \pm 6.7$ & $167.4 \pm 9.3$ \\
Weight $(\mathrm{kg})$ & $71.2 \pm 8.3$ & $73.8 \pm 10.3$ \\
BMI $\left(\mathrm{kg} / \mathrm{m}^{2}\right)$ & $24.0 \pm 1.8$ & $26.3 \pm 2.9$ \\
Glucose $(\mathrm{mg} / \mathrm{dL})$ & $80.6 \pm 8.9$ & $78.9 \pm 10.1$ \\
Cholesterol $(\mathrm{mg} / \mathrm{dL})$ & $163.5 \pm 21.4$ & $143.6 \pm 24.4$ \\
\hline Values are means \pm SD. & There were & no differences \\
between groups. &
\end{tabular}




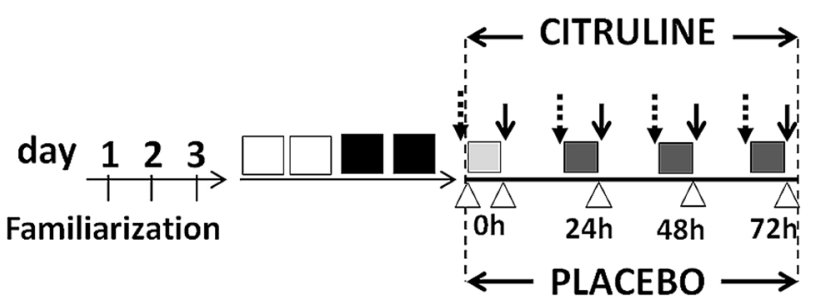

- Leg press 1RM test

- Hack 1RM test

- Resistance training

- Muscular endurance test and electromyography (EMG) signal

$\triangle$ - Blood draw

$\downarrow$ - Perceived exertion - OMNI scale

- Muscle soreness

Figure 1

$119 \times 88 \mathrm{~mm}(300 \times 300 \mathrm{DPI})$ 

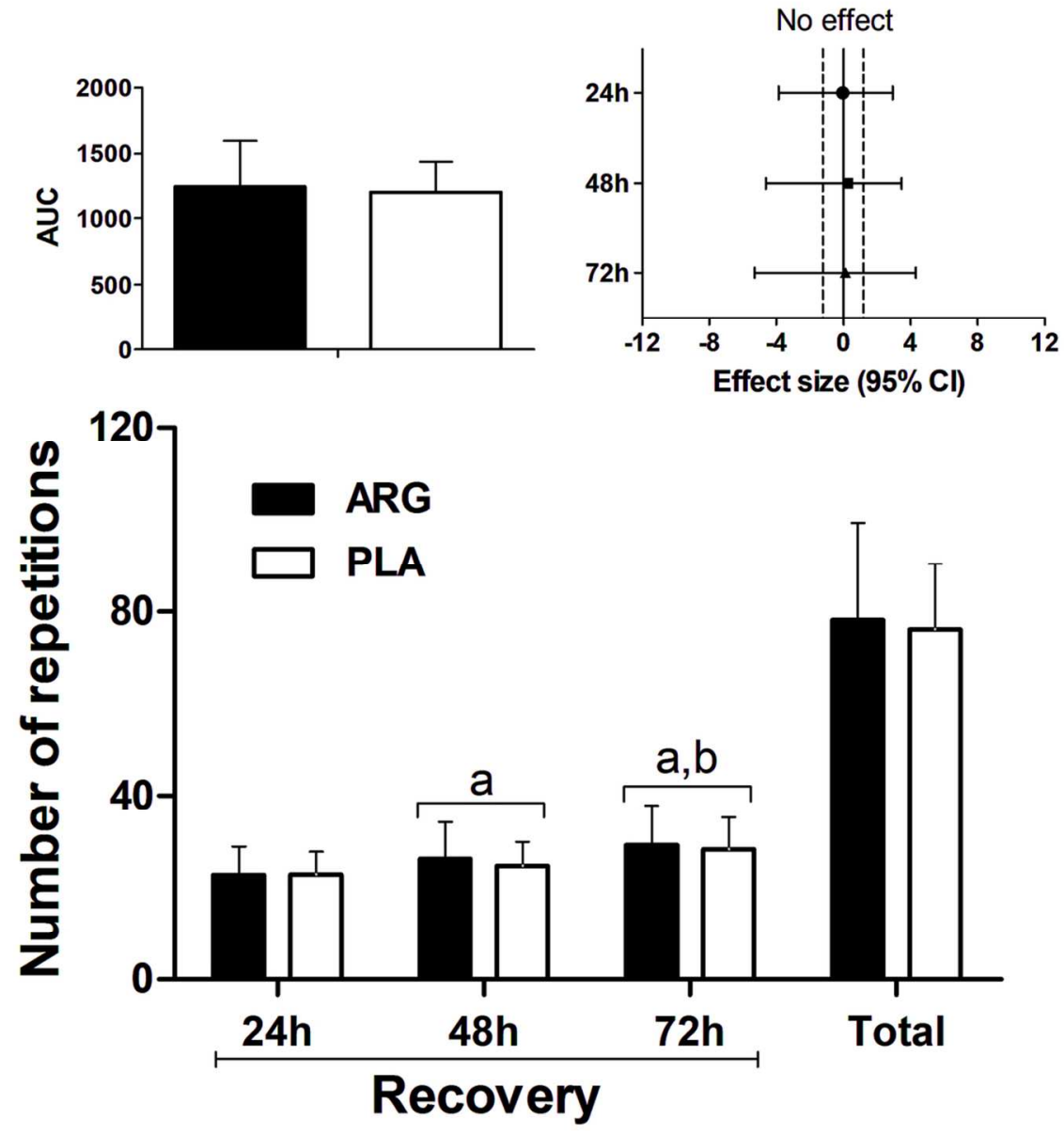

Figure 2

$109 \times 109 \mathrm{~mm}(300 \times 300$ DPI $)$ 

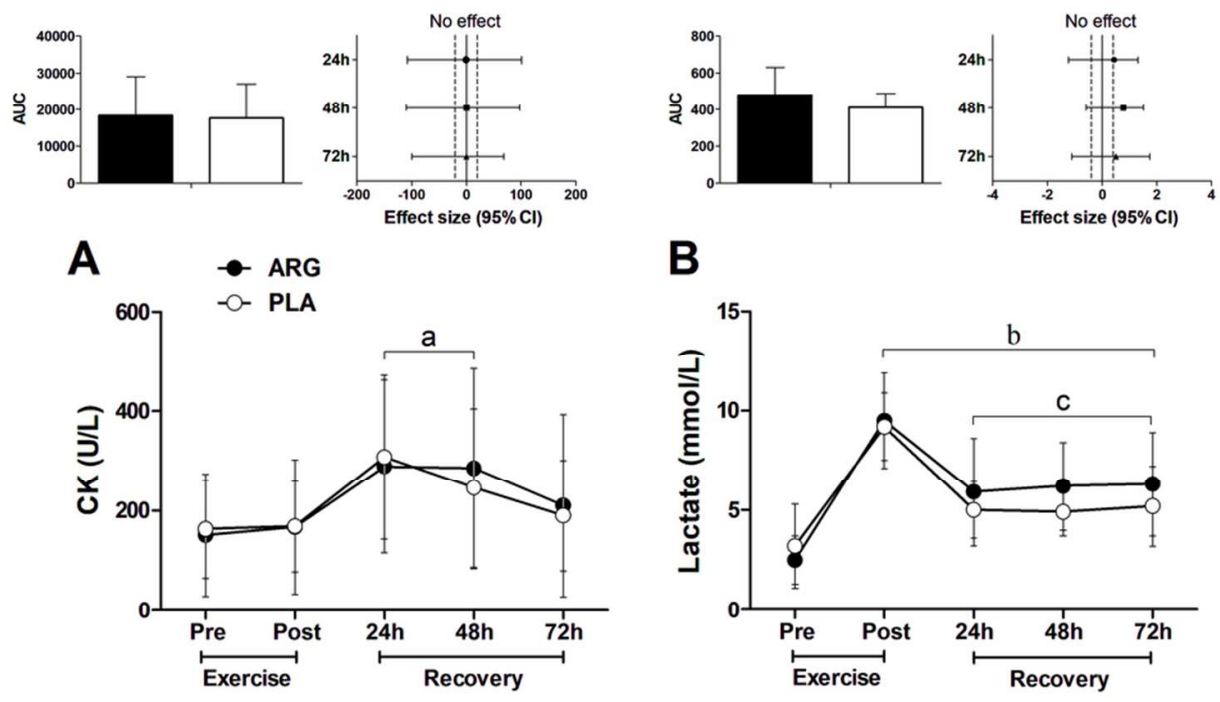

Figure 3

$86 \times 49 \mathrm{~mm}(300 \times 300$ DPI $)$ 

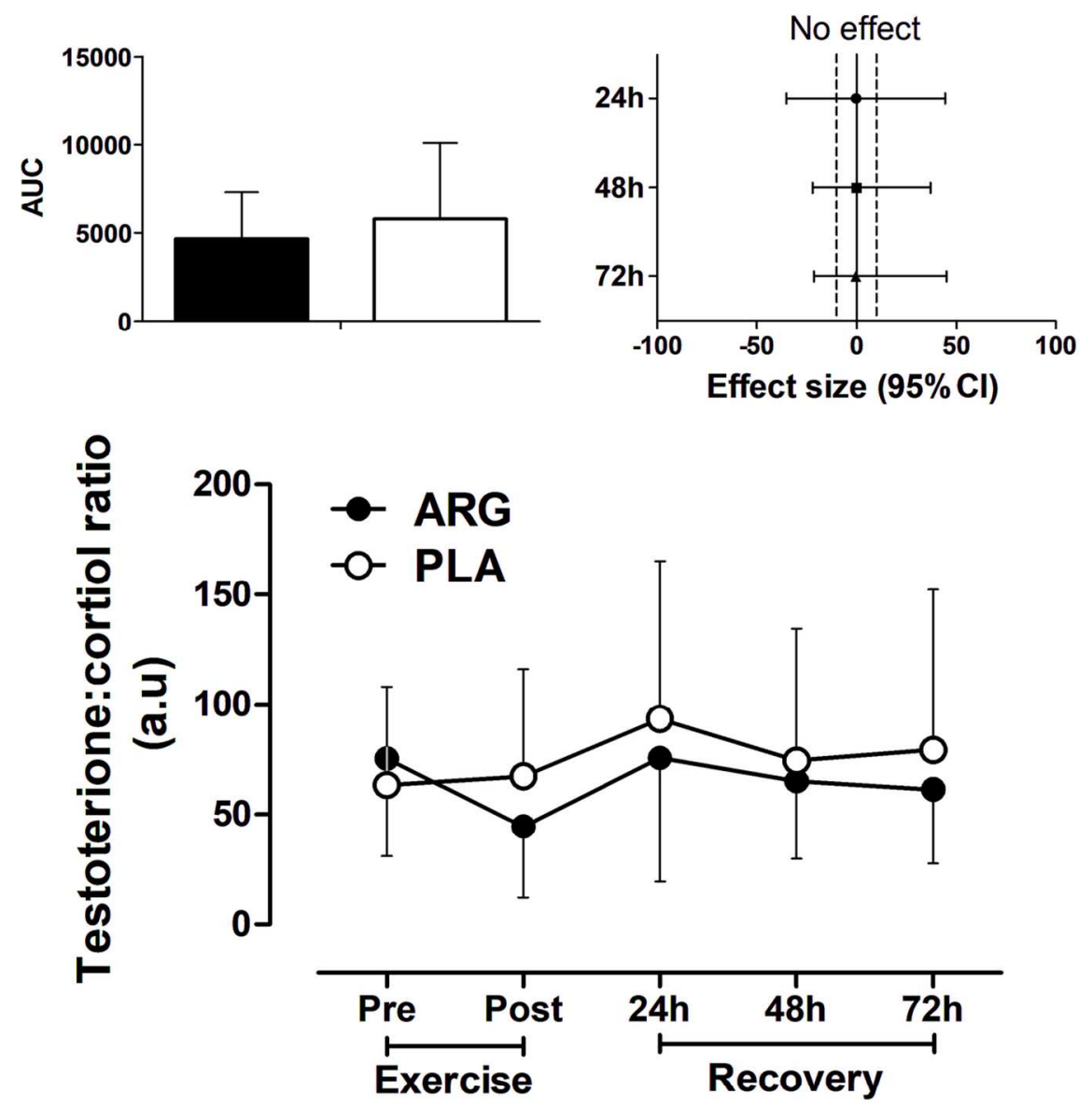

Figure 4

$123 \times 127 \mathrm{~mm}(300 \times 300$ DPI $)$ 

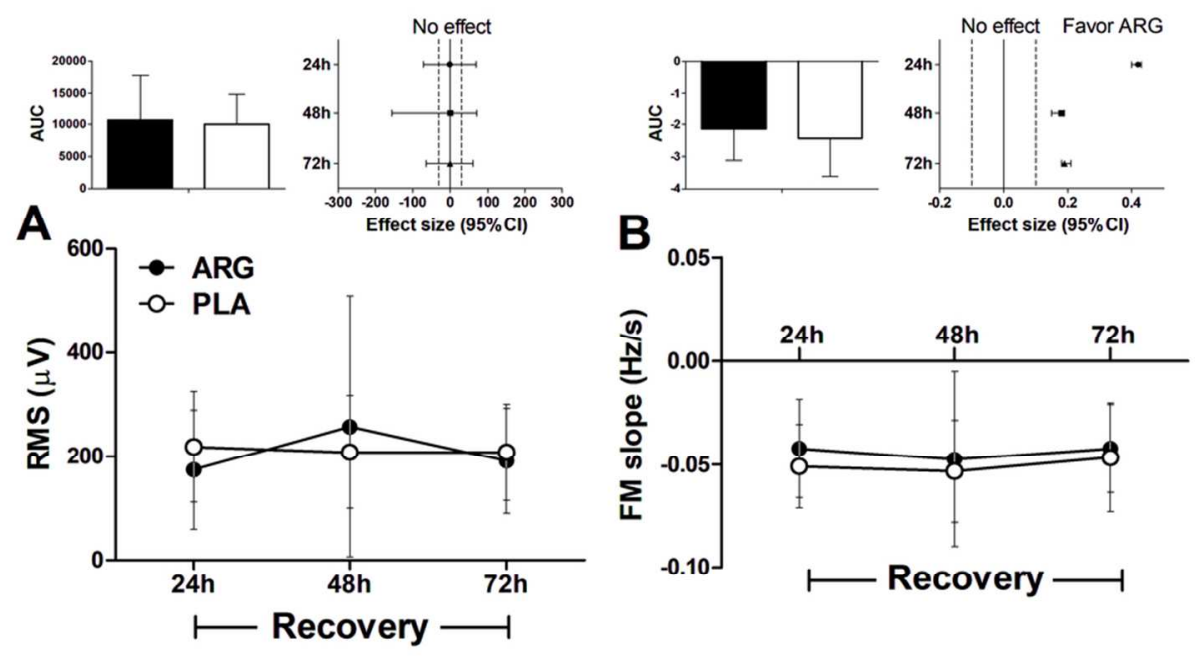

Figure 5

$87 \times 47 \mathrm{~mm}(300 \times 300$ DPI $)$ 